\title{
The impact of different sampling rates and calculation time intervals on ROTI values
}

\author{
Knut Stanley Jacobsen* \\ Norwegian Mapping Authority, Kartverksveien 21, 3511 Hønefoss, Norway \\ *Corresponding author: jacokn@kartverket.no
}

Received 21 August 2014 / Accepted 6 October 2014

\begin{abstract}
The ROTI (Rate of TEC index) is a commonly used measure of ionospheric irregularities level. The algorithm to calculate ROTI is easily implemented, and is the same from paper to paper. However, the sample rate of the GNSS data used, and the time interval over which a value of ROTI is calculated, varies from paper to paper. When comparing ROTI values from different studies, this must be taken into account. This paper aims to show what these differences are, to increase the awareness of this issue. We have investigated the effect of different parameters for the calculation of ROTI values, using one year of data from 8 receivers at latitudes ranging from $59^{\circ} \mathrm{N}$ to $79^{\circ} \mathrm{N}$.

We have found that the ROTI values calculated using different parameter choices are strongly positively correlated. However, the ROTI values are quite different. The effect of a lower sample rate is to lower the ROTI value, due to the loss of high-frequency parts of the ROT spectrum, while the effect of a longer calculation time interval is to remove or reduce short-lived peaks due to the inherent smoothing effect. The ratio of ROTI values based on data of different sampling rate is examined in relation to the ROT power spectrum.

Of relevance to statistical studies, we find that the median level of ROTI depends strongly on sample rate, strongly on latitude at auroral latitudes, and weakly on time interval. Thus, a baseline "quiet" or "noisy" level for one location or choice or parameters may not be valid for another location or choice of parameters.
\end{abstract}

Key words. Algorithm - Space weather - Ionosphere (general) - Irregularities - Total electron content

\section{Introduction}

In the literature, there are many examples of indices used to characterize ionospheric activity. For GNSS (Global Navigation Satellite System) data from normal (not scintillation) receivers, one of the most common indices is the ROTI (Rate of TEC index; Pi et al. 1997). It characterizes small-scale and/ or rapid variations of TEC, and is strongly related to scintillation (Basu et al. 1999). Its main advantage over scintillation indices is that it is calculated based on measurements from normal GNSS receivers, which have been and still are far more common than scintillation receivers. The algorithm to calculate ROTI is easily implemented, and is the same from paper to paper. However, the sample rate of the GNSS data used, and the time interval over which a value of ROTI is calculated, varies from paper to paper. When comparing ROTI values from different studies, this must be taken into account.

Table 1 shows a list of papers in which ROTI is used, and the sampling rate and time intervals used for the ROTI calculations in each of the papers. It is by no means an exhaustive list, but serves as an example that different values are used for those parameters. It can not be said that one choice of parameters is better than another. This depends on how the ROTI is used in each particular case. Problems arise only if comparing the ROTI values from different papers without taking into account the sampling rate and time interval used.

$1 / \mathrm{s}$ and $1 / 30 \mathrm{~s}$ are the most common choices for sampling rates, for the simple reason that these are the two commonly used sample rates for normal GNSS data. Several of the papers discuss the sample rate in relation to the physical phenomenon that is studied in the paper, while other papers note that this was the sampling rate of the data. The choice of sample rate is important for studies of small-scale phenomena, where one must be certain that one can actually measure the phenomena in question. For studies of large-scale phenomena, the sample rate is less important. The time interval can be chosen more freely, as the data files do not dictate what it should be. Xiong et al. (2007) and Carrano \& Groves (2007) chose 1 min intervals to facilitate the comparisons with scintillation indices, which were calculated for 1 min intervals. Krankowski et al. (2006) chose $30 \mathrm{~min}$ intervals in order to cover all or most of a typical patch structure in an interval. Most other papers chose 5 min intervals to get a reasonably fine time resolution, without any specific phenomenon in mind. The choice of time interval is generally a trade-off between time resolution and the quality of the ROTI value, as there should be a good amount of samples per interval. For studies of very large-scale phenomena, it may be advantageous to choose longer time intervals.

The data sources are presented in Section 3. The observations are presented in Section 4 and discussed in Section 5. Finally, Section 6 provides a short summary of our conclusions.

\section{Rate of TEC index}

\subsection{Definition}

ROTI is defined as the standard deviation of the ROT (Rate Of TEC) over some time interval. It is calculated as follows, 
Table 1. Examples of types of ROTI used in literature.

\begin{tabular}{|c|c|c|c|c|}
\hline Reference & $\begin{array}{l}\text { Sampling } \\
\text { rate }\end{array}$ & $\begin{array}{l}\text { Time } \\
\text { interval of } \\
\text { ROTI } \\
\text { calculation }\end{array}$ & Reason for the choice of sampling rate & Reason for the choice of time interval \\
\hline $\begin{array}{l}\text { Pi et al. } \\
\text { (1997) }\end{array}$ & $1 / 30 \mathrm{~s}$ & $5 \mathrm{~min}$ & $\begin{array}{l}1 / 30 \mathrm{~s} \text { was the sampling rate of the data } \\
\text { source used }\end{array}$ & "...allows a relatively high time resolution." \\
\hline $\begin{array}{l}\text { Basu et al. } \\
(1999)\end{array}$ & $1 / 30 \mathrm{~s}$ & $5 \mathrm{~min}$ & $\begin{array}{l}1 / 30 \mathrm{~s} \text { was the sampling rate of the data } \\
\text { source used }\end{array}$ & Refers to Pi et al. (1997). \\
\hline $\begin{array}{l}\text { Beach \& } \\
\text { Kintner } \\
\text { (1999) }\end{array}$ & $\begin{array}{l}\text { Both } 1 / \mathrm{s} \\
\text { and } \\
1 / 30 \mathrm{~s}\end{array}$ & $5 \mathrm{~min}$ & $\begin{array}{l}\text { To compare results using high-resolution and } \\
\text { low-resolution data. }\end{array}$ & $\begin{array}{l}\text { "The basis for a 5-min averaging period is to } \\
\text { permit direct comparison of ROTI with the } \\
\text { results of Pi et al. [1997],..." }\end{array}$ \\
\hline $\begin{array}{l}\text { Krankowski } \\
\text { et al. (2006) }\end{array}$ & $1 / 30 \mathrm{~s}$ & $30 \mathrm{~min}$ & $\begin{array}{l}\text { "This study focuses on the occurrence of } \\
\text { large-scale TEC fluctuations related to patch } \\
\text { structures detected using GPS observations } \\
\text { over Antarctic IGS stations. ... ROT computed } \\
\text { at a } 30-s \text { interval provides data on the } \\
\text { structures on a scale of the order of } \\
\text { kilometers at high latitudes." }\end{array}$ & $\begin{array}{l}\text { "At the size of } 400-1000 \mathrm{~km} \text { and the speed of } \\
\text { the patch structure of } 600-800 \mathrm{~m} / \mathrm{s} \text {, the } \\
\text { duration of TEC eruption recognized the } \\
\text { patch within } 15-30 \text { min. With a view to patch } \\
\text { structure recognition, the index ROT at } \\
30 \text { min intervals was calculated for individual } \\
\text { satellite passes." }\end{array}$ \\
\hline $\begin{array}{l}\text { Xu et al. } \\
(2006)\end{array}$ & $1 / \mathrm{s}$ & $5 \mathrm{~min}$ & $\begin{array}{l}\text { No reason stated in the paper. (But there is } \\
\text { some discussion of scale sizes in the text.) }\end{array}$ & No reason stated in the paper. \\
\hline $\begin{array}{l}\text { Xiong et al. } \\
(2007)\end{array}$ & $1 / 15 \mathrm{~s}$ & $1 \mathrm{~min}$ & $\begin{array}{l}1 / 15 \mathrm{~s} \text { was the sampling rate of the data } \\
\text { source used }\end{array}$ & $\begin{array}{l}\text { "The interval of the } S 4 \text { data is } 1 \text { min." (They } \\
\text { compare ROTI and S4 measurements in the } \\
\text { paper.) }\end{array}$ \\
\hline $\begin{array}{l}\text { Carrano \& } \\
\text { Groves } \\
(2007)\end{array}$ & $\begin{array}{l}10 / \mathrm{s} \text { to } \\
50 / \mathrm{s}\end{array}$ & $1 \mathrm{~min}$ & $\begin{array}{l}\text { "...the fast ROTI parameter is sensitive to } \\
\text { irregularities on the order of } 24 \mathrm{~m} \text {, which is } \\
\text { smaller than the Fresnel scale, and hence to } \\
\text { irregularities that can contribute to amplitude } \\
\text { scintillations at the L1 frequency." }\end{array}$ & $\begin{array}{l}\text { No reason stated in the paper, but it was likely } \\
\text { chosen to be the same as the time resolution } \\
\text { of the scintillation indices. }\end{array}$ \\
\hline $\begin{array}{l}\text { Sripathi } \\
\text { et al. (2011) }\end{array}$ & $1 / 30 \mathrm{~s}$ & $5 \mathrm{~min}$ & $\begin{array}{l}\text { No reason stated in the paper. (But there is } \\
\text { some discussion of scale sizes in the text) }\end{array}$ & Refers to Pi et al. (1997). \\
\hline $\begin{array}{l}\text { Jacobsen \& } \\
\text { Schäfer } \\
(2012)\end{array}$ & $1 / \mathrm{s}$ & $5 \mathrm{~min}$ & $\begin{array}{l}1 / \mathrm{s} \text { was the sampling rate of the data source } \\
\text { used. (But there is some discussion of scale } \\
\text { sizes in the text.) }\end{array}$ & Refers to Pi et al. (1997). \\
\hline
\end{tabular}

where $L n, \lambda_{n}$, and $f_{n}$ are the phase measurement, wavelength, and frequency for the $n$th frequency.

$L_{\mathrm{GF}}(i)$ is the geometry-free phase combination at time $i$

$$
L_{\mathrm{GF}}(i)=L 1(i) \times \lambda_{1}-L 2(i) \times \lambda_{2} .
$$

ROT (in TECU/minute) is calculated as

$$
\operatorname{ROT}(i)=\frac{L_{\mathrm{GF}}(i)-L_{\mathrm{GF}}(i-1)}{\Delta t \times 10^{16} \times 40.3 \times\left(\frac{1}{f_{1}^{2}}-\frac{1}{f_{2}^{2}}\right)} .
$$

TECU (TEC Unit) is defined as $10^{16}$ electrons per $\mathrm{m}^{2} . \Delta t$ is the time difference between the epochs, in minutes. Finally, ROTI, calculated over $N$ epochs, is

$$
\operatorname{ROTI}(i)=\sqrt{\frac{1}{N} \sum_{j=i-N}^{i}(\operatorname{ROT}(j)-\overline{\operatorname{ROT}})^{2}}
$$

\subsection{Drawbacks and limitations}

- The ROTI index does not contain information about the scale size or frequency of the irregularities, only that irregularities exists within the range limited by the sample rate and measurement interval.

- Assuming that the density irregularities do not change within a short time as they pass the receiver-to-satellite line-of-sight, the irregularities may be converted to spatial gradients of TEC (Pi et al. 1997; Yeh \& Liu 1982).
The measurements are related to the spatial scale through the velocity of the plasma relative to the IPP (Ionospheric Pierce Point). The IPP is the intersection of the receiver-to-satellite line with the assumed height of the ionosphere.

As an example, for a relative plasma drift of $1000 \mathrm{~m} / \mathrm{s}$ a ROT measurement of $1 \mathrm{TECU} / \mathrm{min}$ can be converted to $0.017 \mathrm{TECU} / \mathrm{km}$. For a sampling rate of $1 / \mathrm{s}$ and a relative plasma drift of $1000 \mathrm{~m} / \mathrm{s}$, the plasma is sampled every $1000 \mathrm{~m}$. Thus, plasma structures that are smaller than $1 \mathrm{~km}$ may pass by without being detected.

As a rule of thumb, the velocity is on the order of $100 \mathrm{~m} / \mathrm{s}$ at equatorial latitudes and $1000 \mathrm{~m} / \mathrm{s}$ at high latitudes. This must be taken into account when comparing measurements from these two regions.

- The data used to compute ROTI will in most cases not cover the scale sizes required to make a complete comparison to the $\mathrm{S}_{4}$ index (Beach \& Kintner 1999):

"In the IGS network, for example, the sampling rate is 1 sample/30s. As Pi et al. [1997] point out, this rate is usually not sufficient to capture the Fresnel scale, the predominant scale size of weak amplitude scintillation patterns.

For example, irregularities at zenith have a Fresnel scale of $\lambda_{F}=\sqrt{2 \lambda z}$, where $\lambda$ is the free space wavelength of the probing radio signal and $z$ is the altitude. For the 
GPS L1 wavelength of $0.19 \mathrm{~m}$ and $350 \mathrm{~km}$ altitude irregularities, the Fresnel scale is $\lambda_{F}=360 \mathrm{~m}$."

At low latitudes, with drift velocities on the order of $100 \mathrm{~m} / \mathrm{s}$, a $1 / \mathrm{s}$ sampling rate is just barely sufficient to measure fluctuations at that scale size. At high latitudes, with drift velocities an order of magnitude higher, the Fresnel scale is well below the smallest scale size that may be measured using a $1 / \mathrm{s}$ sample rate. In both cases, a 1/30 s sample rate will not measure scale sizes near the Fresnel scale.

It is also noted that the calculation of ROTI requires data from a dual-frequency receiver, whereas the scintillation indices $S_{4}$ and $\sigma_{\phi}$ are calculated using a single frequency (usually the $L 1$ frequency). However, normal dual-frequency receivers are far more numerous than scintillation receivers.

\subsection{Advantages}

- Unlike its interpretation, the calculation of ROTI is quite straightforward.

- As ROTI can be calculated based on data from normal GNSS receivers, there is a lot of data available, with global coverage.

- As the equations for ROTI use the rate-of-change of the geometry-free combination, the biases and ambiguities that often complicate analysis of GNSS data are eliminated.

\subsection{Relation between sampling rate, time interval, and ROTI value}

To make a theoretical prediction about how different sampling rates will affect the ROTI value, we consider the relation of the ROTI value to the power spectrum of the ROT. ROTI is the standard deviation of ROT. The integral over frequency of the power spectrum of ROT is the variance of ROT. The sampling rate determines the upper frequency limit of the integral, while the time interval determines the lower limit. The upper frequency limit is the Nyquist frequency, which is $0.5 \mathrm{~Hz}$ for a $1 / \mathrm{s}$ sampling rate and $\approx 0.0167 \mathrm{~Hz}$ for a $1 / 30$ s sampling rate.

The change in frequency limit from a time interval of $300 \mathrm{~s}$ to a time interval of $3600 \mathrm{~s}$ is $\approx 0.003 \mathrm{~Hz}$. Compared to the change in frequency limit from a sampling rate of $1 / \mathrm{s}$ to a sampling rate of $1 / 30 \mathrm{~s}$, which is $\approx 0.483 \mathrm{~Hz}$, the effects due to different time intervals is expected to be much less significant than the effects due to different sampling rates, with respect to these calculations. The main effect of time interval is then expected to be a smoothing of the signal, which will remove or reduce short-lived peaks while potentially slightly raising the median value.

The theoretical relation between the ROTI values, ignoring the effects of different time intervals, is:

$$
\frac{\operatorname{ROTI}_{X \mathrm{~s}}}{\operatorname{ROTI}_{Y \mathrm{~s}}}=\sqrt{\frac{\int_{v=0}^{1 /(2 * X)} \operatorname{PSD}_{\mathrm{ROT}}(v) \mathrm{d} v}{\int_{v=0}^{1 /(2 * Y)} \operatorname{PSD}_{\mathrm{ROT}}(v) \mathrm{d} v}},
$$

where $\operatorname{PSD}_{\mathrm{ROT}}(v)$ is the power spectral density of ROT at frequency $v$.

To relate the value of ROTI at one sampling rate to the value of ROTI at another sampling rate, we must know the shape of the power spectrum. Making the simplest assumption,

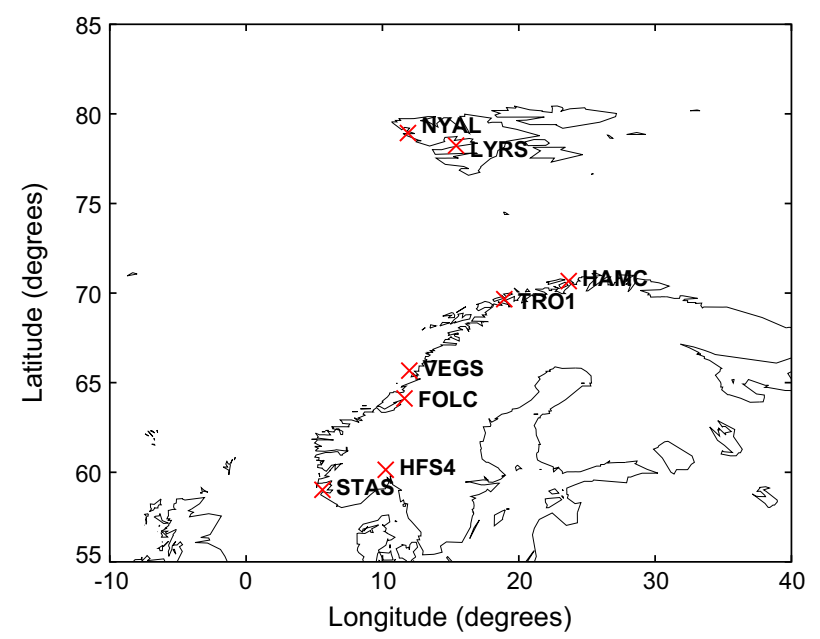

Fig. 1. Geographic locations of the GNSS receivers used in this study.

a level power spectrum, this is the theoretical relation between the ROTI values for 30 s resolution data vs. $1 \mathrm{~s}$ resolution data:

$$
\frac{\mathrm{ROTI}_{30 \mathrm{~s}}}{\operatorname{ROTI}_{1 \mathrm{~s}}}=\sqrt{\frac{\int_{v=0}^{1 / 60} C d v}{\int_{v=0}^{1 / 2} C d v}} \approx 0.18,
$$

where $C$ is an arbitrary constant. (The value of $C$ is not important here, as it is eliminated by the division.)

If the power of the ROT spectrum is enhanced at high frequencies, this ratio will be lower, and if the power of the ROT spectrum is enhanced at low frequencies, this ratio will be higher.

Modeling the TEC as a random walk, or as a random walk plus a linear trend, is one way to produce a level ROT power spectrum, and is a reasonable first approximation to modeling a general TEC time series. How this simple theoretical relation compares to actual observations is explored later in this paper.

\section{Data sources}

This study is based on data from 8 receivers for all of 2012 . Figure 1 shows the locations of the receivers used in this study, and Table 2 lists their basic information. All the receivers are owned and operated by the Norwegian Mapping Authority (NMA). The receivers TRO1 and NYAL also supply data to the International GNSS Service (IGS). All 8 receivers run with a sample rate of $1 \mathrm{~Hz}$, but RINEX files are generated for both $1 / \mathrm{s}$ and $1 / 30 \mathrm{~s}$ sampling rate. The data has been processed to calculate ROTI at various time resolutions (see Table 3 ). This set of parameter values was chosen to illustrate the issue, and does not cover all possible choices of parameter values.

The sampling rates of $1 / \mathrm{s}$ and $1 / 30 \mathrm{~s}$ were chosen because almost all archived GNSS data are recorded at one of these sampling rates. The time interval of $5 \mathrm{~min}$ was chosen because it gives a reasonably good temporal resolution while still having a good number of samples in each interval, and because it is commonly used. The time interval of $1 \mathrm{~h}$ was chosen because it gives a reasonably good number of samples for the $30 \mathrm{~s}$ resolution data. It was used for both $1 / \mathrm{s}$ and $1 / 30 \mathrm{~s}$ data in order to have datasets where the sampling rate was different, but the time interval was the same. The time interval of 
Table 2. List of GNSS receivers.

\begin{tabular}{llccc}
\hline \hline ID & \multicolumn{1}{c}{ Name } & Latitude & Longitude & Type \\
\hline NYAL & Ny-Ålesund & 78.93 & 11.87 & Trimble NetRS \\
LYRS & Longyearbyen & 78.23 & 15.40 & Trimble NetR9 \\
HAMC & Hammerfest & 70.67 & 23.66 & Trimble NetR5 \\
TRO1 & Tromsø & 69.66 & 18.94 & Trimble NetR8 \\
VEGS & Vega & 65.67 & 11.97 & Trimble NetR8 \\
FOLC & Folling & 64.12 & 11.62 & Trimble NetR5 \\
HFS4 & Hønefoss & 60.14 & 10.24 & Trimble NetR5 \\
STAS & Stavanger & 59.02 & 5.60 & Trimble NetR8 \\
\hline
\end{tabular}

Table 3. Parameter values used in this study. The scale size range assumes a relative plasma velocity of $1000 \mathrm{~m} / \mathrm{s}$.

\begin{tabular}{lccc}
\hline \hline Sampling rate & Time interval of ROTI calculation & Scale size range & Samples/interval \\
\hline $1 / \mathrm{s}$ & $5 \mathrm{~min}$ & $1-300 \mathrm{~km}$ & 300 \\
$1 / \mathrm{s}$ & $1 \mathrm{~h}$ & $1-3600 \mathrm{~km}$ & 3600 \\
$1 / 30 \mathrm{~s}$ & $1 \mathrm{~h}$ & $30-3600 \mathrm{~km}$ & 120 \\
$1 / 30 \mathrm{~s}$ & $1 \mathrm{day}$ & $30-86400 \mathrm{~km}$ & 2880 \\
\hline
\end{tabular}

Table 4. Median ROTI values, for geomagnetically quiet days.

\begin{tabular}{lcccc}
\hline \hline ID & 1s 5 min ROTI & 1s 1h ROTI & 30s 1h ROTI & 30s 1 day ROTI \\
\hline NYAL & 0.60 & 0.64 & 0.19 & 0.19 \\
LYRS & 0.56 & 0.61 & 0.05 & 0.15 \\
HAMC & 0.46 & 0.46 & 0.04 & 0.06 \\
TRO1 & 0.45 & 0.45 & 0.03 & 0.05 \\
VEGS & 0.44 & 0.44 & 0.04 & 0.04 \\
FOLC & 0.44 & 0.44 & 0.02 & 0.04 \\
HFS4 & 0.44 & 0.44 & 0.03 & 0.04 \\
STAS & 0.45 & 0.45 & & \\
\hline
\end{tabular}

Table 5. Correlation coefficients and fit parameters for $1 \mathrm{~s} 5$ min ROTI vs. $1 \mathrm{~s} 1 \mathrm{~h}$ ROTI.

\begin{tabular}{lcccc}
\hline \hline ID & Nr of data points & Corr. coeff. & Linear fit offset & Linear fit slope \\
\hline NYAL & 2972 & 0.83 & 0.22 & 0.82 \\
LYRS & 10141 & 0.82 & 0.25 & 0.80 \\
HAMC & 84238 & 0.75 & 0.21 & 0.83 \\
TRO1 & 98734 & 0.76 & 0.17 & 0.85 \\
VEGS & 132703 & 0.79 & 0.06 & 0.90 \\
FOLC & 142661 & 0.78 & 0.07 & 0.90 \\
HFS4 & 169448 & 0.79 & 0.09 & 0.85 \\
STAS & 173693 & 0.76 & & 0.81 \\
\hline
\end{tabular}

1 day was chosen to have a time interval that was significantly longer than $1 \mathrm{~h}$, and to check that the relations between different time intervals still held for such a long time interval. It is not expected that this will be a commonly used time interval for ROTI calculations.

To completely exclude any low elevation issues, an elevation cutoff of $60^{\circ}$ was used.

\section{Observations}

Table 4 shows the median value of ROTI for each parameter choice and each receiver, for geomagnetically quiet days. A geomagnetically quiet day is here defined as a day in which the $K p$ index never exceeded 3. This criterion was matched by 284 days for the time interval investigated here.

The ROTI values calculated with some pairs of parameter choices were compared by making scatter plots, computing the correlation coefficients, and computing the linear fit of one type of ROTI vs. the other type of ROTI.

Table 5 shows the correlation coefficients and fit parameters for $1 \mathrm{~s} 5 \mathrm{~min}$ ROTI vs. $1 \mathrm{~s} 1 \mathrm{~h}$ ROTI. An example of a scatter plot is shown in Figure 2.

Table 6 shows the correlation coefficients and fit parameters for $1 \mathrm{~s} 1 \mathrm{~h}$ ROTI vs. $30 \mathrm{~s} 1 \mathrm{~h}$ ROTI. An example of a scatter plot is shown in Figure 3.

Table 7 shows the correlation coefficients and fit parameters for $30 \mathrm{~s} 1 \mathrm{~h}$ ROTI vs. 30s 1 day ROTI. An example of a scatter plot is shown in Figure 4.

A couple of features in the scatter plots may appear strange:

- The discretization of the values in the lower ends of the scales of the scatter plots, especially noticeable in Figures 3 and 4, is due to the numerical resolution used during the calculations (0.01 TECU/min). 


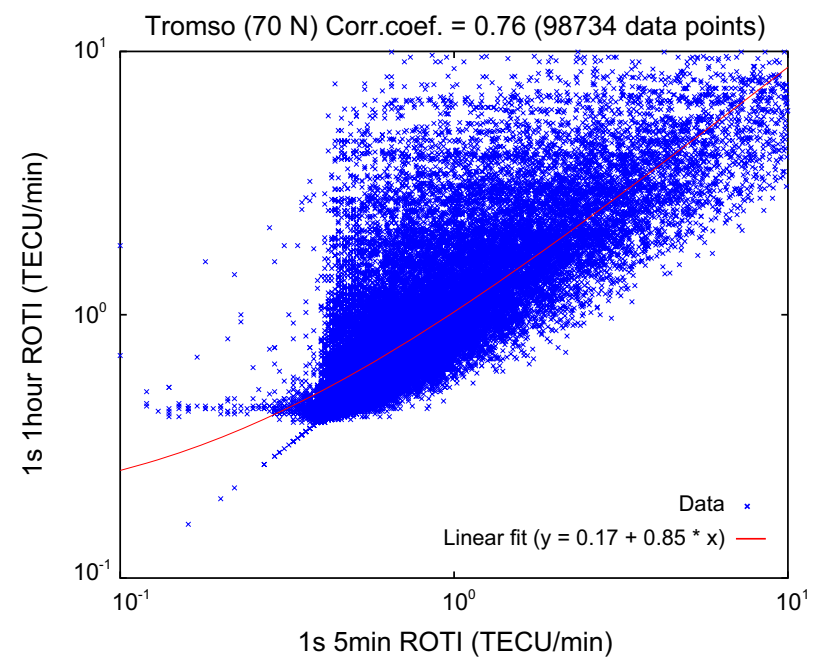

Fig. 2. Scatter plot of $1 \mathrm{~s} 5$ min ROTI vs. $1 \mathrm{~s} 1 \mathrm{~h}$ ROTI. The red line shows a linear fit to the data. Note that the axes of the plot are logarithmic, while the linear fit was performed on the ROTI values before taking their logarithm.

- The "tail" of data points that levels off towards the left edge of the plot in Figure 2. Those seem to be a physical phenomenon in the data, possibly an instrumental source of noise or error. Fortunately, their effect is negligible for two reasons:

They comprise only about $1 \%$ of the data points in the plot.

As they are located in the lower end of a two-decade scale, their influence on the slope parameter of the linear fit is practically zero. The slope parameter is the fit parameter that is the most interesting.

\subsection{Case studies}

This section contains a more detailed look at a few selected days. These case studies are not intended as complete analyses of the events, but as examples of the behavior of ROTI in different situations.

For each day, these types of plots are available:

- TEC spectrogram. These plots show the median TEC spectra for each hour. The spectra are based on 1-h segments of $1 / \mathrm{s}$ samples of TEC. A more detailed description of the calculation is found in the footnote. ${ }^{1}$

- Rate-Of-TEC (ROT) spectrogram. These plots show the median ROT spectra for each hour. The spectra are based on 1-h segments of $1 / \mathrm{s}$ samples of ROT, and calculated in the same way as the TEC spectra.

\footnotetext{
${ }^{1} \operatorname{TEC}_{h}^{s}(t)$ is the time series of TEC for satellite $s$ in the hour-ofday $h$, and consists of up to 3600 TEC samples. $\operatorname{PSTEC}_{h}^{s}(f)$ is the power spectrum of $\operatorname{TEC}_{h}^{s}(t)$ for satellite $s$ in the hour-of-day $h$, as a function of frequency $f$. For each hour, the median spectrum $\operatorname{MPSTEC}_{h}(f)$ is calculated by taking the median at each frequency, over the set of satellites; $\operatorname{MPSTEC}_{h}(f)=\operatorname{median}\left(\operatorname{PSTEC}_{h}^{s}(f)\right)$. Each spectrum $\operatorname{MPSTEC}_{h}(f)$ is one column in the spectrogram plot.
}

- ROTI timeseries, for two kinds of ROTI; $1 \mathrm{~s} 1 \mathrm{~h}$ ROTI and $30 \mathrm{~s} 1 \mathrm{~h}$ ROTI. Each data point shown in the plot is a median of all ROTI values of that type recorded during the hour.

- Normalized ROTI value as a function of sample rate. For these plots, a 1-h segment of $1 \mathrm{~s}$ resolution data was decimated to produce data sets with sample rates from $1 / \mathrm{s}$ to $1 / 300 \mathrm{~s}$. Then the ROTI values were calculated for each decimated data set, and divided by the value of the ROTI from the $1 \mathrm{~s}$ resolution data.

\subsubsection{2-11-09 - Quiet day}

This day was a very quiet day, with $K p \leq 1$ throughout the day and no detected ionospheric disturbances. The TEC and ROT spectrograms (Fig. 8) both show slightly elevated fluctuations in the middle of the day. The ROTI values (Fig. 9, top panel) are low and approximately constant throughout the day.

A 1-h segment of data was processed in more detail. This particular segment was chosen because it had one of the most level ROT power spectrum.

Figure 5 shows the power spectrum of the TEC, along with linear fits to the low-, middle-, and high-frequency parts of the spectrum. The slope in the low and middle parts is $\approx-2$, while the slope in the high-frequency part is -1.5 . These slope values were approximately the same for most of the spectrums that were examined during the work with this paper.

Figure 6 shows the power spectrum of the ROT, along with linear fits to the low-, middle-, and high-frequency parts of the spectrum. The values of the slopes were similar for most of the quiet-day spectrums that were examined during the work with this paper, with values varying within \pm 0.4 .

Figure 7 shows the ROTI value as a function of sample rate.

\subsubsection{2-11-14 - Disturbed day}

This day contains the main part and decline of a geomagnetic storm which reached a level of G2 $(K p=6)$ on the NOAA geomagnetic storm scale (Poppe 2000). Compared to the spectrogram for the quiet day, the TEC spectrogram (Fig. 13) is enhanced for the first part of the day, slowly decreasing until it reaches the quiet-day levels at about 15 UT. During these hours, the ROT spectrogram shows strong enhancements, especially for the first $5 \mathrm{~h}$ of the day. Both of the ROTI values (Fig. 14, top panel) follow the same pattern, starting very high in the early hours of the day and gradually declining until they reach a floor value.

It is noteworthy that at the start of the day, the ROTI values are quite close for the two kinds of ROTI, but as they decline, the decrease for ROTI based on 1/30s data is greater than the decrease for ROTI based on 1/s data. Thus, the ratio between the two kinds of ROTI changes throughout the day.

A 1-h segment of data was processed in more detail. This particular segment was chosen because it had one of the most unequally distributed ROT power spectrum.

Figure 10 shows the power spectrum of the TEC, along with linear fits to the low-, middle-, and high-frequency parts of the spectrum. The shape of the spectrum is similar to the shape of the quiet-day spectrum. 
Table 6. Correlation coefficients and fit parameters for $1 \mathrm{~s} 1 \mathrm{~h}$ ROTI vs. $30 \mathrm{~s} 1 \mathrm{~h}$ ROTI.

\begin{tabular}{lcccc}
\hline \hline ID & Nr of data points & Corr. coeff. & Linear fit offset & Linear fit slope \\
\hline NYAL & 106 & 0.89 & -0.11 & 0.45 \\
LYRS & 281 & 0.74 & -0.01 & 0.30 \\
HAMC & 5997 & 0.95 & -0.12 & 0.44 \\
TRO1 & 6980 & 0.92 & -0.12 & 0.42 \\
VEGS & 10113 & 0.89 & -0.15 & 0.43 \\
FOLC & 10946 & 0.87 & -0.14 & 0.43 \\
HFS4 & 13598 & 0.83 & -0.13 & 0.49 \\
STAS & 14050 & 0.77 & & 0.36 \\
\hline
\end{tabular}

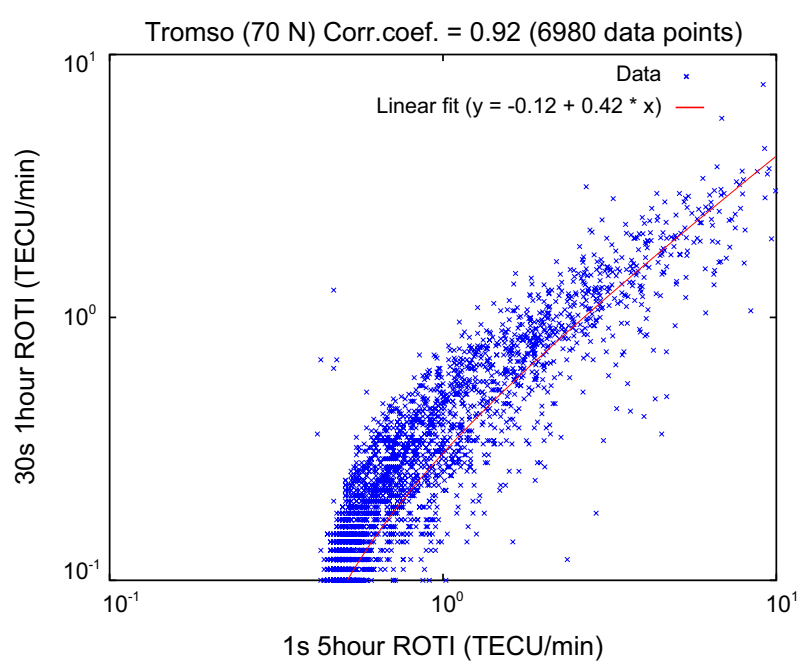

Fig. 3. Scatter plot of $1 \mathrm{~s} 1 \mathrm{~h}$ ROTI vs. $30 \mathrm{~s} 1 \mathrm{~h}$ ROTI. The red line shows a linear fit to the data. Note that the axes of the plot are logarithmic, while the linear fit was performed on the ROTI values before taking their logarithm.

Figure 11 shows the power spectrum of the ROT, along with linear fits to the low-, middle-, and high-frequency parts of the spectrum. The values of the slopes for the disturbedday spectrums that were examined during the work with this paper showed a much greater variation than the slope values for the quiet-day spectrums. Level spectra, spectra with more power at low frequencies, and spectra with more power at high frequencies were all observed. The amount of data investigated in this way is insufficient to determine the relative occurrence rate of the different shapes of spectra.

Figure 12 shows the ROTI value as a function of sample rate.

\section{Discussion}

Table 4 shows the median value of ROTI for each parameter choice and each receiver, for geomagnetically quiet days. This can be seen as the noise level of the ROTI value for days with quiet conditions in the ionosphere. The values are very similar for the same sampling rate at the same receiver, but the values for $1 / 30$ s sampling rate is significantly lower than the values for $1 / \mathrm{s}$ sampling rate.

The median ROTI values are constant with latitude for receivers at latitudes of $59-66^{\circ}$ North, but rises with latitude for receivers at latitudes of $69-79^{\circ}$ North. This is likely due to activity in the auroral oval, which is located at roughly those latitudes during quiet conditions. The increase is not strong enough to cause problems, but is an indication that the quietday level of activity in the auroral ionosphere is slightly higher than at other latitudes. We note that for the 1/s sampling rate data from the two receivers at approximately $70^{\circ}$ North, the increase is almost non-existent, but it is noticeable in their $1 / 30$ s sampling rate data.

Section 4 presented comparisons between different types of ROTI, through scatter plots, correlations and linear fits.

The correlations between ROTI using 1/s sampling rate and different time intervals were strongly positive, with correlation coefficients in the range $0.75-0.83$. The slopes of the linear fits were in the range $0.80-0.90$. The positive offsets seen in Table 5, and the fact that the median values shown in Table 4 were the same or higher for the longer time interval, are consistent with the expected behavior as described in Section 2.4. The calculation at a longer time interval causes a smoothing of short-lived peaks, which simultaneously causes an increase of the median value.

The correlations between ROTI using different sampling rates and a $1 \mathrm{~h}$ time interval were strongly positive, with correlation coefficients in the range $0.74-0.95$. It is noted that the number of data points for NYAL and LYRS was not very high, so the results for those receivers are not as confident as the results from the other receivers. The slopes of the linear fits were in the range $0.30-0.49$. The negative offsets seen in Table 6 , and the fact that the median values shown in Table 4 were far lower for the lower sample rate, are consistent with the expected behavior as described in Section 2.4. The calculation with a lower sample rate includes only a sub-part of the spectrum compared to the calculation at the higher sample rate. This causes the ROTI values from the lower sample rate to be lower. The slopes, however, are greater than the expected value. This is an indication that when disturbances occur, the most common change of the ROT spectrum is that there is a greater increase at lower frequencies of the spectrum. This causes the lower sample rate ROTI to increase more in value than expected relative to the higher sample rate ROTI. An example of this kind of behavior was shown in Section 4.1.2.

The correlations between ROTI using 1/30s sampling rate and different time intervals were strongly positive, with correlation coefficients in the range $0.69-0.86$. The slopes of the linear fits were in the range $0.69-0.92$. The positive offsets seen in Table 7, and the facts that the median values shown in Table 4 were the same or higher for the longer time interval, are consistent with the expected behavior as described in Section 2.4. The calculation at a longer time interval causes a smoothing of short-lived peaks, which simultaneously causes an increase of the median value.

Two days of data were examined in more detail in Section 4.1, and from each of those days a 1-h segment was 
K.S. Jacobsen: Effects of ROTI sampling rates \& time intervals

Table 7. Correlation coefficients and fit parameters for $30 \mathrm{~s} 1 \mathrm{~h}$ ROTI vs. $30 \mathrm{~s} 1$ day ROTI.

\begin{tabular}{lcccc}
\hline \hline ID & Nr of data points & Corr. coeff. & Linear fit offset & Linear fit slope \\
\hline NYAL & 106 & 0.69 & 0.13 & 0.69 \\
LYRS & 281 & 0.81 & 0.07 & 0.92 \\
HAMC & 5997 & 0.86 & 0.06 & 0.86 \\
TRO1 & 6980 & 0.88 & 0.05 & 0.88 \\
VEGS & 10116 & 0.83 & 0.03 & 0.90 \\
FOLC & 10941 & 0.86 & 0.02 & 0.85 \\
HFS4 & 13598 & 0.77 & 0.02 & 0.86 \\
STAS & 14047 & 0.77 & & 0.77 \\
\hline
\end{tabular}

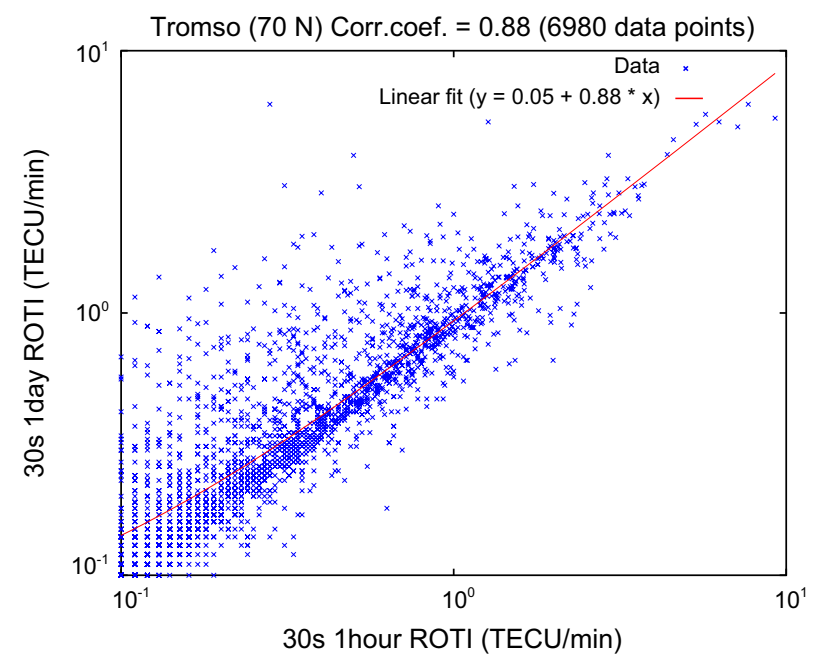

Fig. 4. Scatter plot of $30 \mathrm{~s} 1 \mathrm{~h}$ ROTI vs. $30 \mathrm{~s} 1$ day ROTI. The red line shows a linear fit to the data. Note that the axes of the plot are logarithmic, while the linear fit was performed on the ROTI values before taking their logarithm.

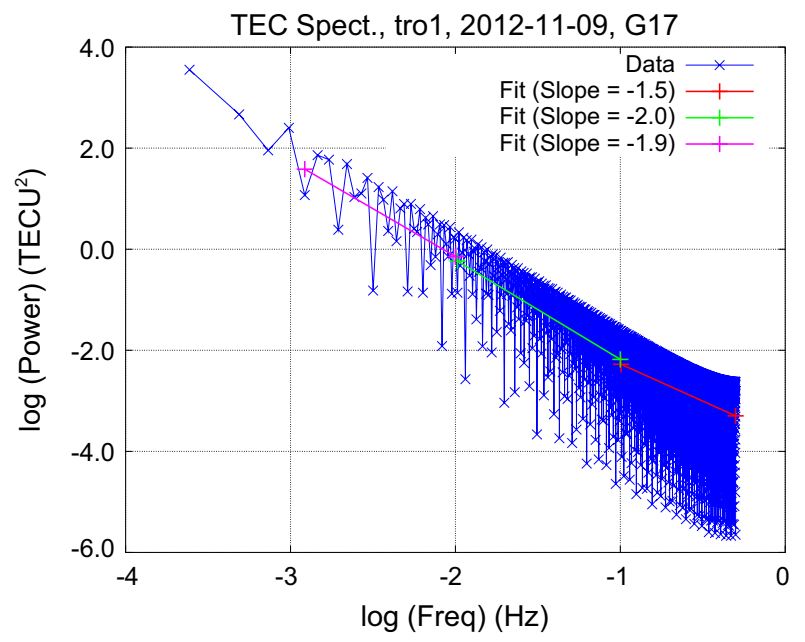

Fig. 5. TEC power spectrum for GPS satellite 17 observed by receiver TRO1. Note that the axes are logarithmic, as indicated by the label text "log (Quantity)".

examined in detail. In particular, the relation between ROTI values calculated based on data of different sample rate (Eq. (4)) was tested. Figures 7 and 12 show the normalized ROTI as a function of sample rate, and the theoretical relation under the assumption of a level ROT spectrum. For the first case, the ROT spectrum was indeed level, and the observed

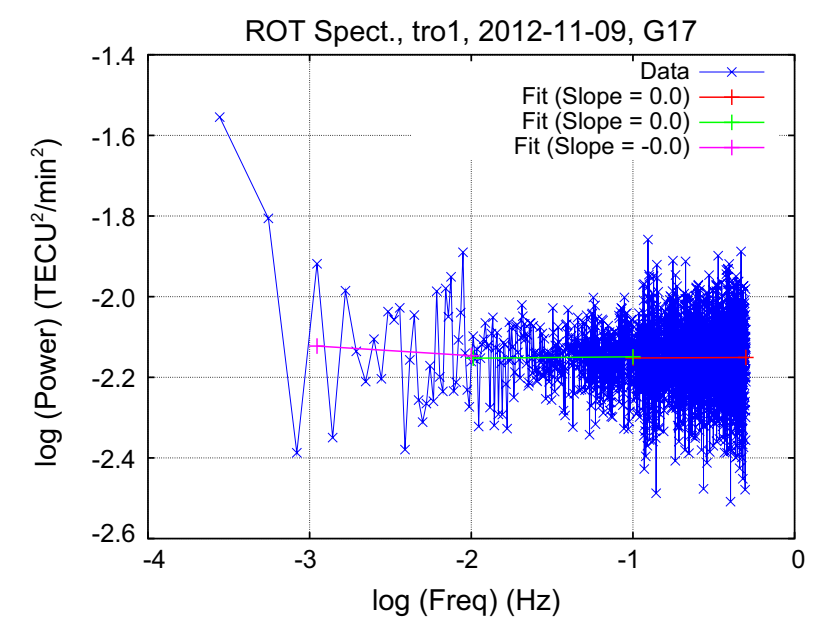

Fig. 6. ROT power spectrum for GPS satellite 17 observed by receiver TRO1. Note that the axes are logarithmic, as indicated by the label text "log (Quantity)".

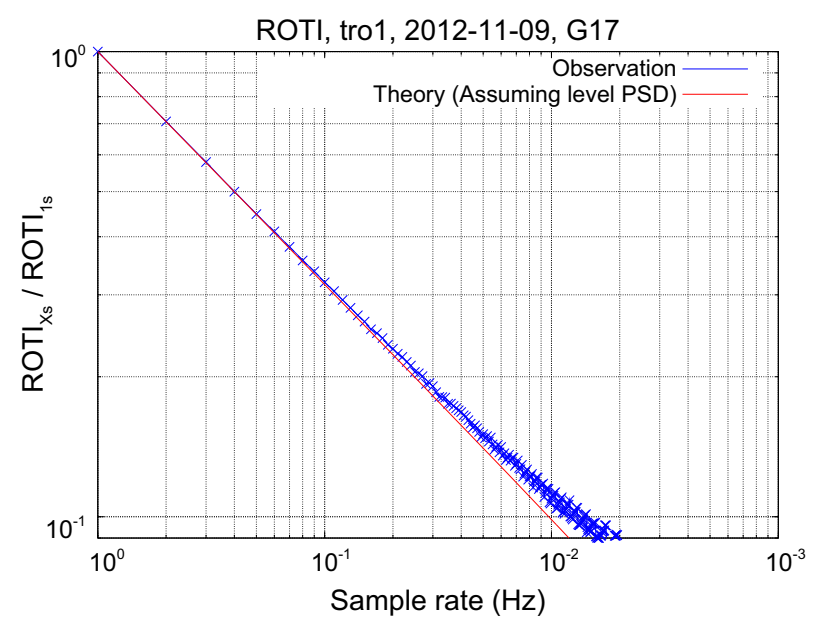

Fig. 7. ROTI as a function of sample rate, normalized to the value for $1 \mathrm{~s}$ resolution data. The red line shows the theoretical value, assuming a level ROT power spectrum.

ROTI values followed the theoretical relation closely. For the second case, the ROT spectrum contained far more power at low frequencies than at high frequencies, and the ROTI did not follow the theory that assumed a level ROT spectrum. As a greater part of the ROT variance was contained at low frequencies, the ROTI value decreased slower with increasing 


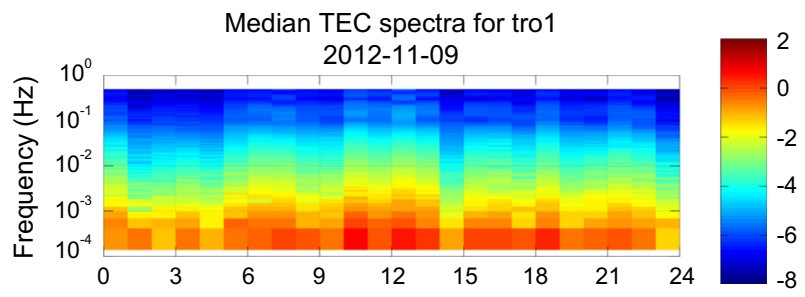

Median ROT spectra for tro1

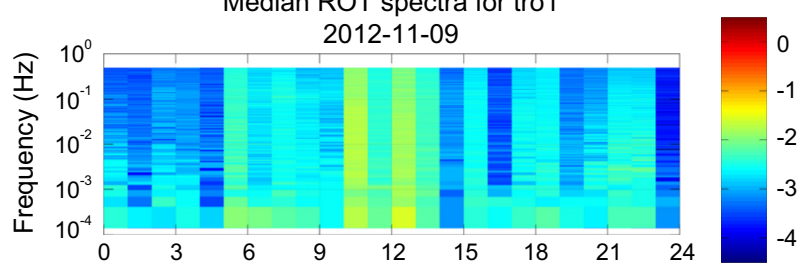

Fig. 8. Median spectra for TEC and ROT throughout the day 2012$11-09$, for receiver TRO1. $X$-axis unit is hour-of-day. $Y$-axis unit is frequency in Hz. Color scale unit is $\mathrm{TECU}^{2}$ for the top panel and $\mathrm{TECU}^{2} / \mathrm{min}^{2}$ for the bottom panel.
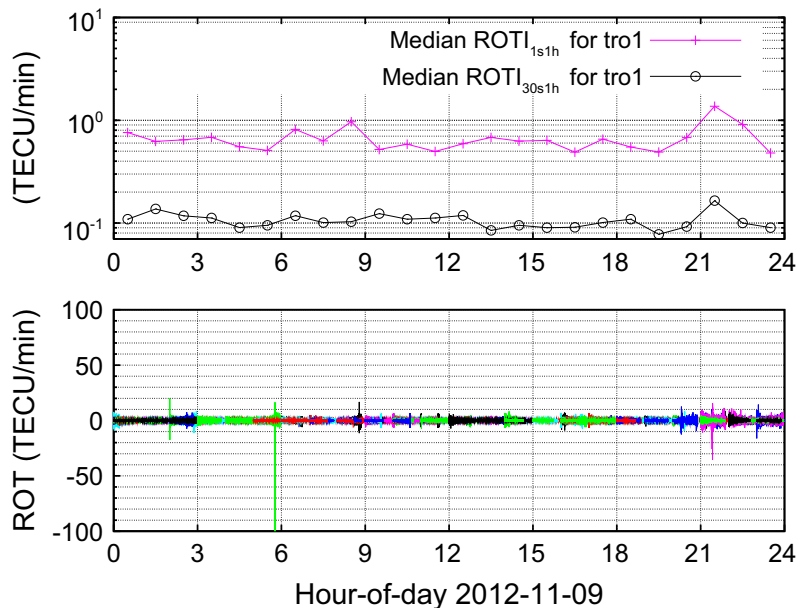

Fig. 9. Top panel: Median hourly ROTI values throughout the day 2012-11-09, for receiver TRO1. Bottom panel: All ROT time series that were used to calculate the median ROTI $_{1 \text { slh }}$ (purple line) in the top panel. Different colors indicate different satellites. Note that each of the colors has been used for several satellites.

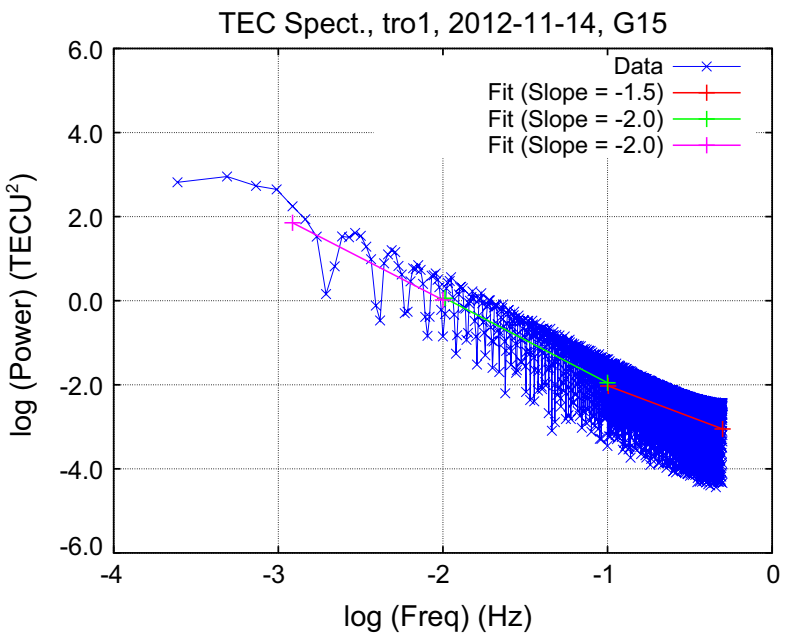

Fig. 10. TEC power spectrum for GPS satellite 15 observed by receiver TRO1. Note that the axes are logarithmic, as indicated by the label text "log (Quantity)".

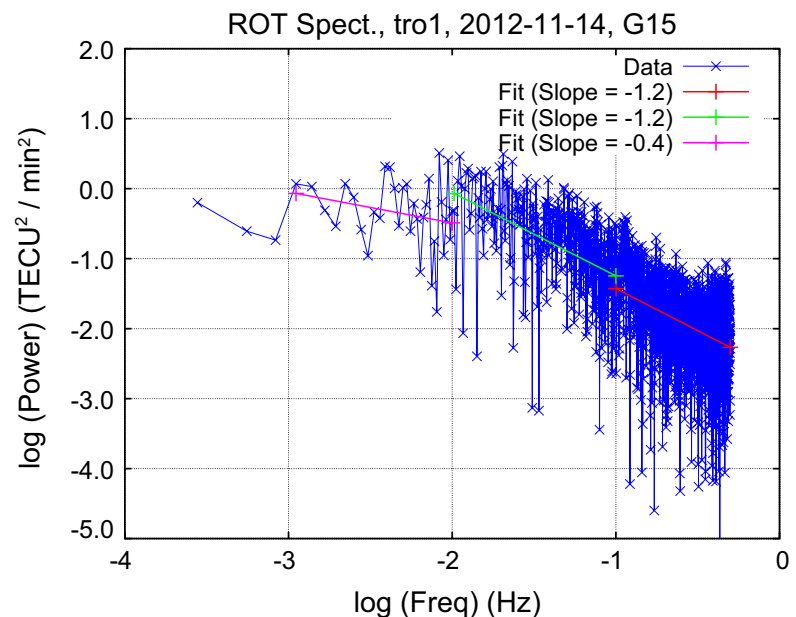

Fig. 11. ROT power spectrum for GPS satellite 15 observed by receiver TRO1. Note that the axes are logarithmic, as indicated by the label text "log (Quantity)".

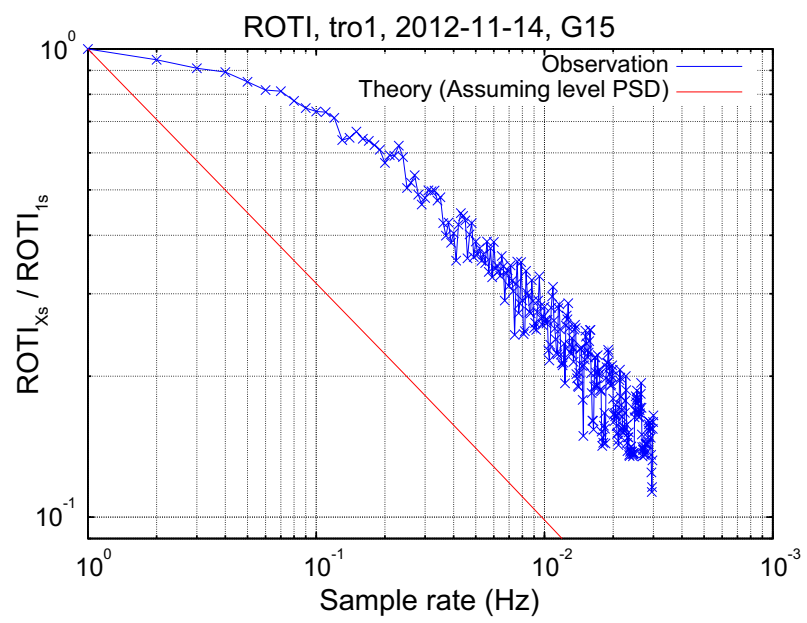

Fig. 12. ROTI as a function of sample rate, normalized to the value for $1 \mathrm{~s}$ resolution data. The red line shows the theoretical value, assuming a level ROT power spectrum.

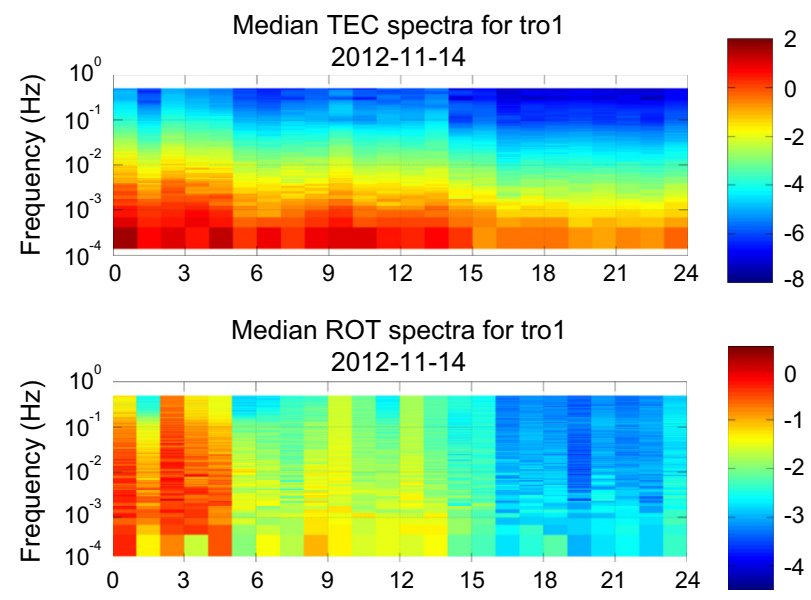

Fig. 13. Median spectra for TEC and ROT throughout the day 2012-11-14, for receiver TRO1. $X$-axis unit is hour-of-day. $Y$-axis unit is frequency in $\mathrm{Hz}$. Color scale unit is $\mathrm{TECU}^{2}$ for the top panel and $\mathrm{TECU}^{2} / \mathrm{min}^{2}$ for the bottom panel. 

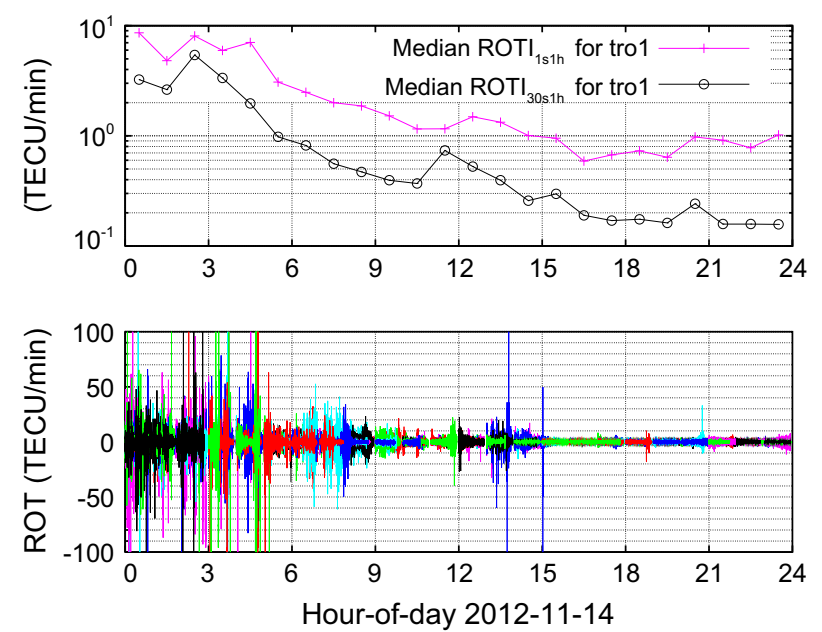

Fig. 14. Top panel: Median hourly ROTI values throughout the day 2012-11-14, for receiver TRO1. Bottom panel: All ROT time series that were used to calculate the median $\mathrm{ROTI}_{1 \mathrm{sih}}$ (purple line) in the top panel. Different colors indicate different satellites. Note that each of the colors has been used for several satellites.

sample rate. As the sample rate decreased, the upper frequency limit came closer to the break point in the ROT spectrum, and the slope of ROTI vs. sample rate approached the slope of the line that assumed a level spectrum.

\section{Conclusions}

We have investigated the effect of different parameters for the calculation of ROTI values. These are our conclusions:

- The effect of a lower sample rate is to lower the ROTI value, due to the loss of high-frequency parts of the ROT spectrum. If the ROT spectrum is level, the decrease will follow the relation given in Eq. (4), but it has been observed that the ROT spectra are not necessarily level during disturbed conditions. We have found indications that during disturbed conditions, the low-frequency part of the ROT spectrum most often increases more than the high-frequency part of the spectrum. Detailed studies that use different sets of parameters for ROTI calculation should take the shape of the ROT spectrum into account.

- The effect of a longer calculation time interval is to remove or reduce short-lived peaks due to the inherent smoothing effect. Depending on the number and size of peaks, this may cause a noticeable increase in the median value of ROTI. This should be kept in mind when comparing different statistical studies using ROTI.

- At high latitudes $\left(\gtrsim 70^{\circ}\right.$ North), the median value of ROTI is higher than at lower latitudes. This should be taken into account when defining ROTI limits for a study or system using data from those latitudes.

- As the median level of ROTI depends strongly on sample rate, strongly on latitude at auroral latitudes, and weakly on time interval, a baseline "quiet" or "noisy" level for one location or choice or parameters may not be valid for another location or choice of parameters. If a baseline is not available for the location and set of parameters used in a study, a large amount of data should be analyzed to determine a baseline before sorting the ROTI into categories such as "quiet" and "noisy".

It is noted that these conclusions are based on data from receivers at latitudes $\geq 59^{\circ}$ North. They may not be applicable at equatorial latitudes. Also, these results may not be valid for ROTI calculation parameters greatly outside the range investigated here (see Table 3).

Acknowledgements. $K p$ index data was downloaded from the NOAA/National Geophysical Data Center (NGDC) file server. The author thanks the reviewers for their helpful comments. The editor thanks Iurii Cherniak and two anonymous referees for their assistance in evaluating this paper.

\section{References}

Basu, S., K. Groves, J. Quinn, and P. Doherty. A comparison of TEC fluctuations and scintillations at Ascension Island. J. Atmos. Sol. Terr. Phys., 61 (16), 1219-1226, 1999,

DOI: 10.1016/S1364-6826(99)00052-8, http://www.sciencedirect. com/science/article/pii/S1364682699000528.

Beach, T.L., and P.M. Kintner. Simultaneous Global Positioning System observations of equatorial scintillations and total electron content fluctuations. J. Geophys. Res. [Space Phys.], 104 (A10), 22,553-22,565, 1999, DOI: 10.1029/1999JA900220.

Carrano, C.S., and K. Groves. TEC Gradients and Fluctuations at Low latitudes Measured with High Data Rate GPS Receivers. Proceedings of the 63rd Annual Meeting of the Institute of Navigation, Cambridge, MA, 156-163, 2007.

Jacobsen, K.S., and S. Schäfer. Observed effects of a geomagnetic storm on an RTK positioning network at high latitudes. J. Space Weather Space Clim., 2, A13, 2012,

DOI: $10.1051 / \mathrm{swsc} / 2012013$.

Krankowski, A., I. Shagimuratov, L. Baran, I. Ephishov, and $\mathrm{N}$. Tepenitzyna. The occurrence of polar cap patches in TEC fluctuations detected using GPS measurements in southern hemisphere. Adv. Space Res., 38 (11), 2601-2609, 2006, Middle and Upper Atmospheres, Active Experiments, and Dusty Plasmas, DOI: 10.1016/j.asr.2005.12.006, http://www.sciencedirect. com/science/article/pii/S0273117705014225.

Pi, X., A.J. Mannucci, U.J. Lindqwister, and C.M. Ho. Monitoring of global ionospheric irregularities using the worldwide GPS network. Geophys. Res. Lett., 24 (18), 2283-2286, 1997, DOI: 10.1029/97GL02273.

Poppe, B.B. New scales help public, technicians understand space weather. Eos, Transactions American Geophysical Union, 81 (29), 322-328, 2000, DOI: 10.1029/00EO00247.

Sripathi, S., B. Kakad, and A. Bhattacharyya. Study of equinoctial asymmetry in the Equatorial Spread F (ESF) irregularities over Indian region using multi-instrument observations in the descending phase of solar cycle 23. J. Geophys. Res. [Space Phys.], 116 (A11), 2011, DOI: 10.1029/2011JA016625.

Xiong, B., W.-X. Wan, B.-Q. Ning, H. Yuan, and G.-Z. Li. A Comparison and analysis of the $\mathrm{S} 4$ index, $\mathrm{C} / \mathrm{N}$ and Roti over Sanya. Chinese J. Geophys., 50 (6), 1414-1424, 2007, DOI: $10.1002 / \mathrm{cjg} 2.1161$.

Xu, J.-S., J. Zhu, and G.-H. Chen. GPS observations of ionospheric effects of the major storm of Nov. 7-10, 2004. Chinese J. Geophys., 49 (4), 848-855, 2006, DOI: 10.1002/cjg2.904.

Yeh, K.C., and C.-H. Liu. Radio wave scintillations in the ionosphere. Proc. IEEE, 70 (4), 324, 1982. 